VIII $^{\text {èmes }}$ Journées Nationales Génie Civil - Génie Côtier, Compiègne, 7-9 septembre 2004

\title{
Rôle des crues dans la production de sédiments transportés en suspension dans un cours d'eau des bassins versants méditerranéens
}

\author{
A. Megnounif, A. Terfous, A. Ghenaim, J.B. Poulet \\ ERESA, INSA de Strasbourg \\ a.ghenaim@insa-strasbourg.fr
}

\section{Résumé:}

Le processus du transport des matières solides en suspension dans les bassins versants est complexe. Il est étroitement lié à l'intensité des précipitations, à la configuration du bassin versant et aux conditions hydrauliques. Dans ce contexte et pour mieux comprendre l'influence de ces facteurs sur la production et le cheminement des particules en suspension dans les cours d'eau, on s'est intéressé à l'Oued Sebdou, cours d'eau principal drainant le bassin versant de la Haute-Tafna, situé au nord-ouest algérien. Pour ce faire, on étudie, pour les différents épisodes de crue, le mode d'évolution de la charge solide en fonction des débits liquides en s'appuyant sur l'analyse des hystérésis.

\section{Abstract:}

The process of the transport of suspended matter in watershads is strictly connected to the intensity of the precipitation, to the configuration of catchment and to the hydraulic conditions. In this context and to understand the influence of these factors on the production and the progress of particles in the Upper-Tafna watershad, situated in the northwest of Algeria. To do it, we study the relationships between discharge and suspended sediment concentration during hydrologic events by leaning on the analysis of hysteresis.

Mots-clés: crue, sédiment, suspension, hystérésis, Méditerranéen, Algérie.

Keywords: flood, sediment, suspension, hysteresis, Mediterranean.

\section{Introduction}

Le Maghreb septentrional est l'une des régions les plus vulnérables, au monde, à l'érosion hydrique. Walling ${ }^{1}$ estime les dégradations spécifiques dans ces régions entre 1000 et 5000 tonnes par $\mathrm{km}^{2}$ et par an. Probst et Amiotte - Suchet ${ }^{2}$ montrent que ces dégradations sont très variables et peuvent atteindre comme pour le cas de l'Oued Agrioun en Algérie 7200 tonnes par $\mathrm{km}^{2}$ et par an. Comme conséquence de ces dégradations, la part des sédiments qui se déverse annuellement dans la mer Méditerranée est estimée à 100 millions de tonnes.

Outre les pertes en terres fertiles ainsi que l'alluvionnement et l'exhaussement des fonds de barrages, des ports et estuaires, les sédiments en suspension constituent un support de nombreux contaminants chimiques provenant des bassins versants ${ }^{3}$. Comprendre le cheminement des particules en suspension, c'est aussi comprendre le devenir de nombreux contaminants.

Il est connu que le transport des matières en suspension se fait principalement en période de crue et que les principaux facteurs qui contrôlent la production et le transport en suspension dans les bassins versants sont dus essentiellement à l'effet conjugué de l'intensité des précipitations, la configuration du bassin versant et les caractéristiques hydrauliques du cours d'eau. 
Dans ce contexte, on sintéresse au bassin versant de la Haute-Tafna (256 km²). On analysera le mode d'évolution de la charge solide $\mathrm{C}$ en fonction des débits liquides $\mathrm{Q}_{\mathrm{L}}$ durant les différents événements de crues. Ce genre d'étude à été introduit initialement par Wood ${ }^{4}$ pour étudier les événements de crues dans un bassin versant. Plus tard, Williams ${ }^{5}$ et Asselman ${ }^{6}$ présentent une classification détaillée des différents types d’hystérésis représentants l'évolution des crues dans les rivières des Etats-Unis.

\section{$\underline{\text { 2.Présentation du site }}$}

Situé au nord ouest algérien, le bassin versant de la Haute-Tafna est encaissé dans les massifs de l'Atlas tellien (fig.1). Il s'étale sur une superficie de $256 \mathrm{~km}^{2}$, et se caractérise par un relief abrupt. 49\% de la superficie du bassin présente des pentes supérieures à 25\%. Le couvert végétal est pauvre et discontinu. Le cours d'eau principal Oued Sebdou s'écoule sur une longueur de $27 \mathrm{~km}$. Il se creuse dans des formations essentiellement gréseuses sur lesquelles se sont développées des formations carbonatées bien karstifiées ${ }^{7}$. Le nord et le nord-est du bassin versant correspondent à un horst jurassique principalement carbonaté. Au sud et à l'est on trouve un graben rempli de sédiments plio-quaternaires représentant le fossé de Sebdou.

Sur la région règne un climat fort contrasté, qui, par bien de ses aspects est agressif. Les hauts massifs se caractérisent par un climat froid et pluvieux. Alors que sur les plaines, situées de part et d'autre des chaînes montagneuses de l'Atlas, règne un climat relativement sec à fort écart thermique, ce qui détermine la sécheresse estivale excessive engendrant des orages violents.

Le régime d'écoulement est de type bi-modal à influence méditerranéenne avec deux maxima, un principal autour du mois de mars et un secondaire en novembre. Le minimum estival est plus faible que celui de la période hivernale. Toutefois, selon les années, les valeurs mensuelles maximales peuvent s'inter changer ${ }^{8,9}$.

\section{Source de données et méthode d'étude}

Dans le cadre des transferts de matières solides en suspension dans le bassin versant de la Haute-Tafna, on dispose de valeurs instantanées de débits liquides en $\mathrm{m}^{3} / \mathrm{s}$ et de turbidités exprimées en g/l, fournies par l’A.N.R.H., «Agence Nationale des Ressources Hydriques» et mesurées sur 5 années hydrologiques de septembre 1988 à août 1993.

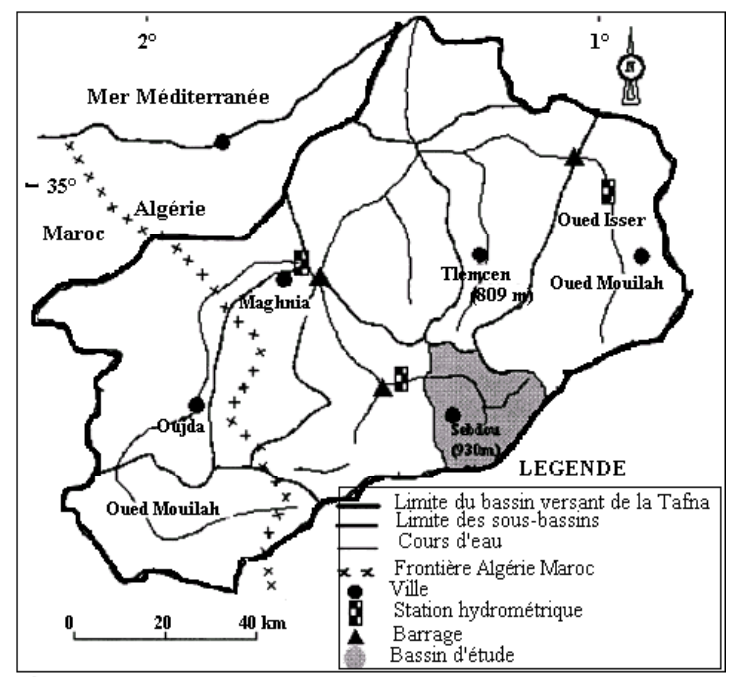

Figure 1 Situation du bassin versant de la Haut-Tafna. 
Le transfert des matières solides dans les cours d'eau est étroitement lié à la façon dont sont distribuées les précipitations sur la surface du bassin versant, à l'importance relative des divers composants de l'écoulement les uns par apport aux autres (ruissellement superficiel, ruissellement différé et contribution des écoulements a partir des réservoirs souterrains), à la diversité des matériaux en taille et en nature, ainsi qu'aux contraintes dynamiques des particules solides dans les cours d'eau (capacité de dépôt ou de remise en suspension ou encore de maintien en suspension).

Dans ce contexte et pour comprendre le cheminement des particules transportées en suspension dans le cours d'eau de l'Oued Sebdou, on étudie les hystérésis représentant l'évolution de la charge solide en fonction du débit liquide à la chronique des événements durant le cycle hydrologique. Les crues prises en considération sont celles qui se manifestent par une variation notable du débit liquide, et dépassent le module annuel.

\section{Résultats et discussion}

La charge en suspension transportée par les cours d'eau pérennes est en général étroitement liée aux débits liquides suivant un modèle en puissance. En ce qui concerne les cours d'eau à écoulement temporaire cette relation reste très modérée ${ }^{10,11}$. En effet, pour l'Oued Sebdou, le nuage de points est relativement dispersé (fig. 2). La dispersion des points est due essentiellement à la particularité des événements.

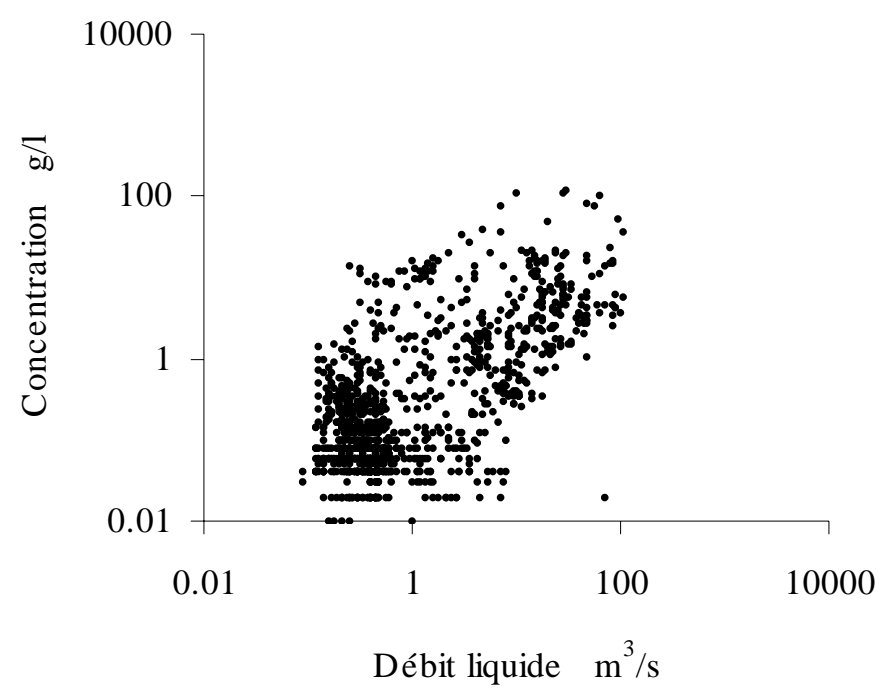

Figure 2:Variation de la concentration des matières solides en suspension en fonction du débit liquide (septembre 1988 - août 1993).

Au cours du cycle hydrologique, les périodes de crues jouent un rôle prédominant dans l'exportation des matières en suspension. En effet, c'est durant les épisodes de crues que les apports solides dans les cours d'eau sont très significatifs. Les apports en eau et en matières solides transportées en suspension par l'Oued Sebdou, (tab. I) montrent les résultats suivants :

- La contribution des crues dans l'apport annuel en eau est estimée en moyenne à 64\%, sur un apport total de l'ordre de 30,9 millions de $\mathrm{m}^{3}$. Alors que le flux des matières solides transportées en suspension par ces dernières constitue la quasi-totalité, soit 93\%, du flux annuel moyen évalué à 286000 tonnes.

- La charge moyenne annuelle des eaux ramenées par l'Oued Sebdou est de 9,3 g/l. Restreintes aux épisodes des crues, cette charge passe à 13,6 g/l. Elle est très variable d'une 
année à une autre, variant de moins de $1 \mathrm{~g} / \mathrm{l}$ pour l'année 1992-93, à près de 20 g/l pour l’année 1988-89.

D’autre part, l'enregistrement simultané des débits liquides $\mathrm{Q}_{\mathrm{L}}$ en $\mathrm{m}^{3} / \mathrm{s}$ et des turbidités en g/l (fig. 3), permet de souligner les résultats suivants :

- La charge solide en suspension augmente avec les débits liquides sans qu'il y ait une véritable proportionnalité entre ces deux paramètres. En effet, les plus fortes teneurs en MES atteintes 115 et $105 \mathrm{~g} / \mathrm{l}$ ont été enregistrées respectivement suite à un orage estival survenu en juillet 1989 et aux pluies automnales enregistrées en septembre 1990. Alors que, les débits liquides extrêmes observés sont respectivement 30 et $106 \mathrm{~m}^{3} / \mathrm{s}$.

Tableau 1 Bilan annuel des apports liquides $\left(A_{L}\right.$ en million de $\left.m^{3}\right)$, et solides ( $A_{S}$ en million de tonnes), et contribution des crues. (septembre 1988 à août 1993)

\begin{tabular}{|c|c|c|c|c|c|c|c|}
\hline \multirow[b]{2}{*}{ Année } & \multicolumn{3}{|c|}{ Apport liquide } & \multicolumn{3}{|c|}{ Apport solide } & \multirow{2}{*}{\begin{tabular}{|c|} 
Charge des \\
eaux de crue \\
$(\mathrm{g} / \mathrm{l})$
\end{tabular}} \\
\hline & $\begin{array}{l}\text { Total } \\
\left(\mathrm{Mm}^{3}\right)\end{array}$ & $\begin{array}{l}\text { Crue } \\
\left(\mathrm{Mm}^{3}\right)\end{array}$ & $\begin{array}{l}\text { Contr. } \\
(\%)\end{array}$ & $\begin{array}{l}\text { Total } \\
(\mathrm{M} \mathrm{T})\end{array}$ & $\begin{array}{l}\text { Crue } \\
(\mathrm{MT})\end{array}$ & $\begin{array}{c}\text { Contr. } \\
(\%)\end{array}$ & \\
\hline $1988-89$ & 23,7 & 14,0 & 59 & 0,274 & 0,271 & 99 & 19.6 \\
\hline 1989-90 & 10,9 & 3,4 & 31 & 0,014 & 0,009 & 67 & 2.6 \\
\hline 1990-91 & 77,9 & 62,4 & 80 & 1,094 & 1,009 & 92 & 16.2 \\
\hline 1991-92 & 26,7 & 14,6 & 55 & 0,041 & 0,038 & 93 & 2.6 \\
\hline 1992-93 & 15,1 & 4,3 & 29 & 0,006 & 0,004 & 61 & 0.9 \\
\hline Moyenne & 30,9 & 19,7 & 64 & 0,286 & 0,266 & 93 & 13.6 \\
\hline
\end{tabular}

Durant les cinq années de mesures, 4 à 5 crues au plus sont enregistrées le long de chaque cycle hydrologique. Ces épisodes de crues alternent avec des périodes d'étiages sévères, voire jusqu'à rupture de l'écoulement. Le temps moyen séparant deux crues consécutives a été estimé à 62 jours. Cet intervalle peut être long et dépasser les 5 mois.

Les crues sont concentrées en épisodes qui durent quelques jours, et dont la somme ne représente, en moyenne, que $12 \%$ du temps annuel.

Au cours du cycle hydrologique, une à deux crues au plus constituent l'essentiel des apports liquides et solides.

La charge solide en été est plus importante que celle transportée par les crues printanières, pour deux raisons. D’une part, les pluies estivales sont généralement intenses et plus agressives, et d'autre part, le couvert végétal plus abondant au printemps peut limiter le splash et empêcher le ruissellement.

La dynamique de l'état hydrique affecte la structure du sol, ses propriétés hydriques et détermine en grande partie sa susceptibilité à l'érosion. En effet, la cohésion des agrégats est l'une des propriétés importante qui influence l'érosion mécanique des sols. Cependant les mécanismes de désagrégation sont divers et permettent de décomposer l'agrégat en particules isolées de taille plus petite qui pourront être facilement transportées vers le cours d'eau. Une analyse synthétique de l'évolution de la charge solide en fonction des débits liquides suivant les différents épisodes de crue, a permis de distinguer différentes caractéristiques de ces crues.

1).Dans un premier cas, on observe des hystérésis dites rétrogrades, appelées aussi horlogiques. C’est le cas des crues du 18 mars 1989 et du 3 janvier 1991 (fig. 4-a et b). Ce type d'hystérésis révèle un accroissement rapide des concentrations par rapport aux débits liquides, dû en bonne partie à la remise en suspension des dépôts fins dans le lit fluvial et sur les banquettes latérales. Pour la crue du 18 mars 1989 (fig.4-a), la hausse du débit liquide, suite à la fonte des neiges, accroît les paramètres hydrodynamiques dans le lit du cours d'eau. 
Ce qui d'une part, favorise le remaniement des dépôts des particules fines qui s’y étaient formés et d'autre part, érode les berges du cours d'eau.

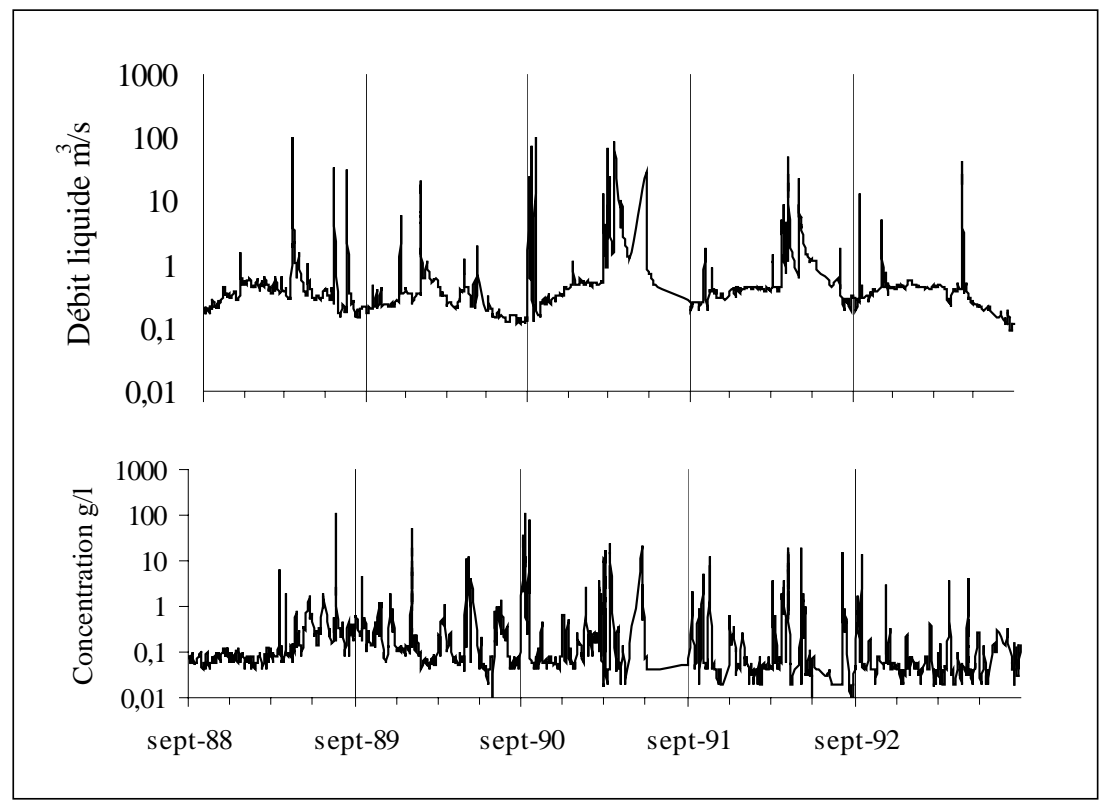

Figure 3. Variation des débits liquides instantanés et des turbidités.

L'action érosive porte essentiellement sur le réseau hydrographique. Dans le deuxième cas de figure (fig. 4-b), la crue est survenue après une sécheresse excessive. En effet, et depuis la longue saison sèche estivale, aucune crue notable n’a été enregistrée avant le 3 janvier 1990. L’impact prolongé des gouttes de pluies sur le sol desséché pulvérise les agrégats de la matrice fine sur les versants produisant des matériaux susceptibles d'être amené au cours d'eau par ruissellement superficiel. La première montée des eaux qui suit les premières pluies permet d'évacuer les particules fines produites par le bassin versant et de chasser les dépôts fins piégés dans les pentes douces du cours d'eau.

Ces crues sont généralement importantes en apport en eau, et surviennent après une longue période d'étiage. Elles correspondent à une longue séquence pluvieuse répartie sur l'ensemble du bassin versant. La charge solide en suspension est importante lors de la montée des eaux. Elle est diluée, lors de la décrue, par les écoulements retardés hypodermiques et souterrains, ce qui permet un curage des particules fines.

2).Dans d'autres cas, l'évolution de la charge solide en suspension en fonction des débits liquides suit un sens antihorlogique et présente des hystérésis dites orthogrades. Ce mode d'évolution se manifeste par un décalage entre l'onde de crue qui affecte les masses d'eau et le transfert plus lent des matériaux fournis à partir des versants. Ces crues sont généralement générées par de grosses averses qui trouvent un sol humide. L'eau présente dans le sol avant la crue, permet d'augmenter les forces de cohésion du sol et diminue le taux de détachement des particules ${ }^{12,13}$. La source principale de la charge solide en suspension est alimentée par l'érosion en ravines et la dégradation des berges du cours d'eau. C'est le cas de la crue du 21 juillet 1989 (fig.4-c). La charge maximale (115,7 g/l) est atteinte une heure après le débit de pointe $30 \mathrm{~m}^{3} / \mathrm{s}$. C'est aussi le cas de la crue du 02 septembre 1990 (fig. 4-d), le décalage entre le débit de pointe $\left(23,5 \mathrm{~m}^{3} / \mathrm{s}\right)$ et la charge maximale $(35,5 \mathrm{~g} / \mathrm{l})$, survenue après, est de deux heures. 


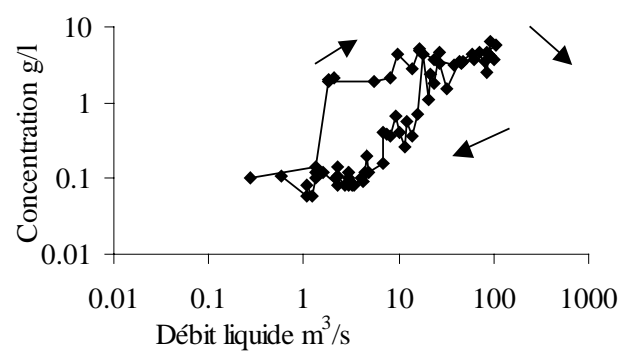

a) Crue du 18 mars 1989

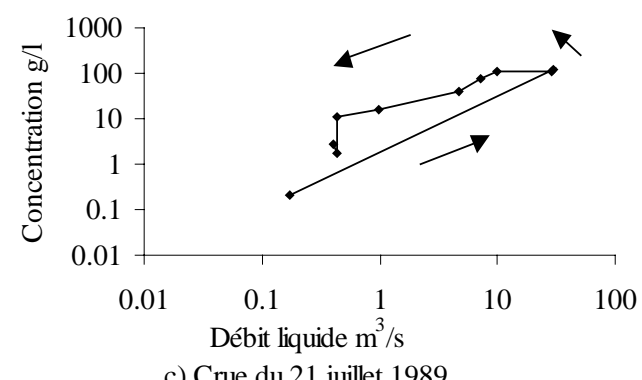

c) Crue du 21 juillet 1989

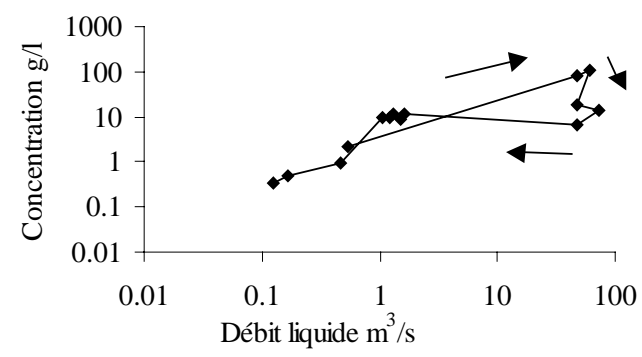

e) Crue du 07 septembre 1990

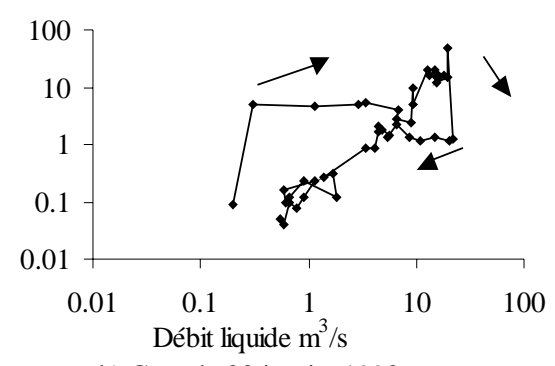

b) Crue du 03 janvier 1990

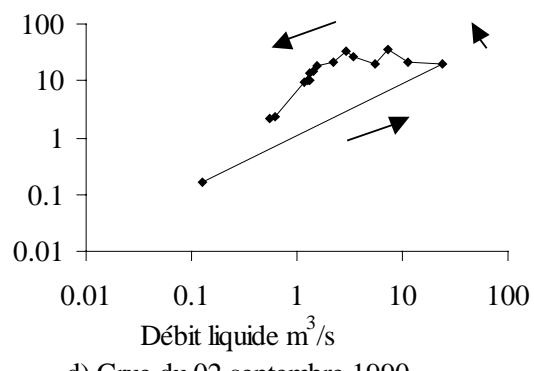

d) Crue du 02 septembre 1990

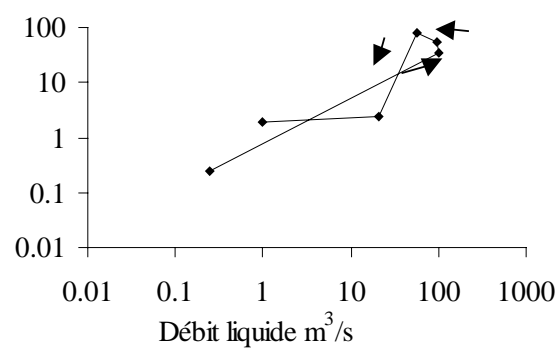

f) Crue du 16 septembre 1990

Figure 4 Mode d'évolution de la charge solide en suspension en fonction des débits liquides pour différents épisodes de crues.

3).Par ailleurs, selon l'intensité des précipitations et la contribution des écoulements retardés, les hystérésis orthogrades et réthrogrades sont différentes de par leurs représentations ouvertes ou serrées. Pour le reste des crues, il y a absence d'hystérésis soit par absence de dépôts fins remobilisables, soit par insuffisance des débits liquides pour évacuer les stocks particulaires déposés, soit encore parce que la crue en question est générée par une succession d'averses de différentes intensités.

\section{Conclusion}

Cette étude a pu mettre en évidence le rôle des crues dans la genèse et le transport des sédiments en suspension dans un petit bassin versant Méditerranéen, le bassin de la HauteTafna. Les résultats obtenus montrent que le transport des matières solides en suspension se fait principalement durant les épisodes de crues. En effet, sur un apport annuel moyen en eau, estimé à 30,9 millions de $\mathrm{m}^{3}$, les crues contribuent par 64\%. Cependant, elles transportent la quasi-totalité, soit 93\%, de la charge solide en suspension évaluée en moyenne annuelle à 286 000 tonnes. 


\section{Références}

1.Walling D.E., (1984). The sediment yields of African rivers. IAHS Publ., 144, 265-283.

2.Probst J.L., Amiotte - Suchet P., (1992). Fluvial suspended sediment transport and mechanical erosion in the Maghreb (North Africa). Hydrol.Sci. J., 37, 621-637.

3.Konhauser K.O., Powell M.A., Fyee W.S., Longstaffe F.J., Tripathy S., (1997). Trace élément geochemistery of river sédiment, Orisa Satate, India. Journal of Hydrology , 193,258269.

4.Wood P.A., (1977). Sediment transport in the Hope River, Jamaïca: a tropical drainage basin characterized by seasonal flow. I.A.H.S. Publ., 22, 149-156.

5.Williams G.P., (1989). Sédiment concentration versus waterdischarge during single hydrologic events in rivers. J. Hydrol., 111, 89-106.

6.Asselman N.EM., (1999). Suspended sediment dynamics in large drainage basin : the River Rhine. Hydrologycal Processes, 13, 1437-1450.

7.Benest M., Elmi S., (1987). Précisions stratigraphiques sur le Jurassique inférieur et moyen de la partie méridionale des Monts de Tlemcen (Algérie). Comptes Rendus Symposium Société Géologie. France, Fasc. 8, 295-296.

8.Megnounif A., Terfous A., Bouanani A., (2003). Production et transport des matières solides en suspension dans le bassin versant de la Haute-Tafna (Nord Ouest Algérien). Rev. Sci. Eau 16(3), 369-380.

9.Terfous A., Megnounif A., Bouanani A., (2001). Etude du transport solide en suspension dans l'Oued Mouilah (Nord Ouest Algérien). Rev. Sci. Eau 14(2), 175-185.

10.Sibari H., Haidi S., Ait fora A., (2001). Typologie des crues et érosion mécanique dans un bassin versant de zone semi-aride : bassin versant de l'Inaouène, Maroc. Science et changements planétaires / Sécheresse, 12(3), 187-193.

11.Alexandrov Y. Laronne J.B., (2003). Suspended sediment transport in flash floods of the semiarid northern Negev, Israel. IAHS Publ., 278, 346-352.

12.Le bissonais Y, Le souder C., (1995). Mesurer la stabilité structurale des sols pour évaluer leurs sensisbilité à la battance et à l'érosion. Etude et Gestion des sols, 2(1), 43-56.

13.Roose E, (1977). Erosion et ruissellement en Afrique de l'Ouest : 20 ans de mesures en petites parcelles. ORSTOM Paris, Travaux et Doc, 78, 150p. 